\title{
Enhancement of the magnetic anisotropy of nanometer-sized Co clusters: Influence of the surface and of interparticle interactions
}

\author{
F. Luis, ${ }^{1}$ J. M. Torres, ${ }^{1}$ L. M. García, ${ }^{1}$ J. Bartolomé,${ }^{1, *}$ J. Stankiewicz, ${ }^{1}$ F. Petroff, ${ }^{2}$ F. Fettar,${ }^{2, \dagger}$ \\ J.-L. Maurice, ${ }^{2}$ and A. Vaurès ${ }^{2}$ \\ ${ }^{1}$ Instituto de Ciencia de Materiales de Aragón, CSIC-Universidad de Zaragoza, 50009 Zaragoza, Spain \\ ${ }^{2}$ Unité Mixte de Physique CNRS/THALES, Domaine de Corbeville, 91404 Orsay, France \\ and Université Paris-Sud, 91405 Orsay, France
}

(Received 30 July 2001; published 6 February 2002)

\begin{abstract}
We study the magnetic properties of spherical Co clusters with diameters between $0.8 \mathrm{~nm}$ and $5.2 \mathrm{~nm}$ (25-7000 atoms) prepared by sequential sputtering of $\mathrm{Co}$ and $\mathrm{Al}_{2} \mathrm{O}_{3}$. The particle size distribution has been determined from the equilibrium susceptibility and magnetization data and it is compared with previous structural characterizations. The distribution of activation energies has been independently obtained from a scaling plot of the ac susceptibility. Combining these two distributions we have accurately determined the effective anisotropy constant $K_{\text {eff }}$. We find that $K_{\text {eff }}$ is enhanced with respect to the bulk value and that it is dominated by a strong anisotropy induced at the surface of the clusters. Interactions between the magnetic moments of adjacent layers are shown to increase the effective activation energy barrier for the reversal of the magnetic moments. Finally, this reversal process is shown to proceed classically down to the lowest temperature investigated $(1.8 \mathrm{~K})$.
\end{abstract}

DOI: 10.1103/PhysRevB.65.094409

PACS number(s): 75.50.Tt, 75.70.-i, 75.40.Gb, 75.70.Rf

\section{INTRODUCTION}

Single-domain magnetic particles are attractive for applications in data storage. Their properties differ from those of the bulk magnets ${ }^{1}$ because, as the size of the particles decreases, an increasing fraction of the total magnetic atoms lies at the surface. The electronic and magnetic structure of these atoms can be modified by the smaller number of neighbors as compared to the bulk ${ }^{2-4}$ and/or by the interaction with the surrounding atoms of the matrix where the particles are dispersed. It was shown that surface effects might lead to a smaller net magnetic moment of the particle. ${ }^{5,6}$ By contrast, experiments performed on "bare" particles of $\mathrm{Fe}, \mathrm{Co}$, and $\mathrm{Ni}$ produced in beams ${ }^{7,8}$ show that the net magnetic moment per atom increases as the size of the cluster decreases, approaching the limiting value for a free atom. In addition, the net anisotropy of the particle exceeds the bulk value., ${ }^{910}$ This excess was recently correlated to the augmentation of the orbital magnetic moment of the peripheral atoms. ${ }^{11,12}$

Magnetic nanoparticles are also good candidates for the study of quantum effects in intermediate scales between the microscopic and the macroscopic classical world. ${ }^{13,14}$ In real systems, however, we usually deal with macroscopic ensembles of particles with different sizes and shapes. The average magnetic properties of these systems come from intraparticle as well as interparticle phenomena, which are usually difficult to disentangle. Therefore, in this field of research it is desirable to obtain systems in which each of the parameters, such as the average particle size, the particle size distribution, the crystalline structure, and the spatial arrangement of the particles, can be varied independently of each other.

We believe that the work reported here is a step forward in this direction. We present the magnetic characterization of a new type of systems of Co nanoparticles, embedded in an amorphous matrix of $\mathrm{Al}_{2} \mathrm{O}_{3}$, prepared by sequential deposition of both materials. ${ }^{15-17}$ By varying the deposition time, the diameter of the aggregates can be controlled between below $1 \mathrm{~nm}$ and $7 \mathrm{~nm}$. For a range of thicknesses, a relatively ordered disposition of the particles with a rather homogeneous dispersion of the particles inside the matrix is obtained, in which they are arranged in layers separated from the adjacent ones by a controllable distance. ${ }^{17}$ The paper is organized as follows. In the first two sections we briefly describe the method employed to prepare the samples and their physical characterization. Then, we present our experimental results. Using the data obtained from ac magnetic susceptibility, zero-field-cooled (ZFC) and field-cooled (FC) magnetization measurements, and isotherms of magnetization as a function of the field, we have determined the particle size distribution in samples which have been prepared with different Co deposition times. We compare these results with available data from a previous structural characterization. This important information is then used to accurately determine the effective anisotropy constant and its variation with the size of the particles. We have also been able to separate surface anisotropy effects from the effect of the dipole-dipole interaction between the magnetic moments of the particles. The last section is left for the conclusions.

\section{MORPHOLOGY AND STRUCTURE OF THE SAMPLES}

The Co aggregates were prepared in Orsay by sputter deposition of Co atoms on a smooth alumina surface. Details of the sample preparation and of its structural characterization have already been reported elsewhere. ${ }^{15-17}$ The amount of deposited Co is given here by the nominal thickness $t_{C o}$ that the deposits would have if they were homogeneous. This amount was measured by using energy-dispersive $\mathrm{x}$-ray spectroscopy in the transmission electron microscope and found to be within less than $5 \%$ of the planned dose in all 
TABLE I. Parameters of the Gaussian distribution of particle's sizes obtained by TEM (a) and from the fit of the magnetization data (b) The width $\sigma$ of the distribution is given in units of the average diameter. The last column gives the estimated fraction of Co atoms which do not aggregate in particles.

(a)

\begin{tabular}{lccccc}
${ }_{t_{C o}}(\mathrm{~nm})$ & $\langle D\rangle^{a}(\mathrm{~nm})$ & $\sigma^{a}$ & $\langle D\rangle^{b}(\mathrm{~nm})$ & $\sigma^{b}$ & $x_{\text {para }}$ \\
\hline 0.1 & & & $0.8(1)$ & $0.35(5)$ & $0.7(2)$ \\
0.2 & $0.83(20)$ & 0.3 & $1.3(1)$ & $0.3(1)$ & $0.7(2)$ \\
0.3 & $1.4(3)$ & 0.3 & $1.4(1)$ & $0.32(5)$ & $0.22(4)$ \\
0.4 & $1.4(3)$ & 0.22 & $2.2(1)$ & $0.2(1)$ & $0.5(1)$ \\
0.7 & $2.9(6)$ & 0.23 & $3.1(3)$ & $0.2(1)$ & $0.25(5)$ \\
1 & $4.2(8)$ & 0.27 & $5.2(3)$ & $0.25(5)$ & $0.13(3)$ \\
\hline \hline
\end{tabular}

cases. Clusters are formed below the percolation limit which appears to occur at $t_{C o}=2 \mathrm{~nm}$. On top of each Co layer a new alumina layer of about $3 \mathrm{~nm}$ was deposited. Oxidized $\mathrm{Si}$ was used as a substrate. A given sample is usually made by piling up a number $N$ (1-100 for the samples studied here) of these layers. ${ }^{17}$ It was found that the amount of Co deposited on the surface is larger than the Co mass which forms clusters visible by transmission electron microscopy (TEM). The relative difference between these quantities increases as $t_{C o}$ decreases. Therefore, we have in our samples nonaggregated atoms or very small clusters, which contribute to the magnetic signal of the samples, in addition to Co aggregates. One of the difficulties of the interpretation of the magnetic data is to separate these two contributions.

The morphology, size, and spatial distribution of the aggregates were also studied using the TEM data. The aggregates are of approximately spherical shape (at least for $t_{C o}$ $\langle 1 \mathrm{~nm}$ ). The average diameter $\langle D\rangle$ of the particles increases linearly with $t_{C o}$. We give in Table I a list of the important parameters obtained from these experiments for all samples studied.

\section{EXPERIMENTAL DETAILS}

The magnetic measurements were performed in Zaragoza using a commercial Superconducting quantum interference device (SQUID) magnetometer in the temperature range of $1.8 \mathrm{~K}<T<320 \mathrm{~K}$ and in magnetic fields up to $5 \mathrm{~T}$. The ac susceptibility was measured by applying a small ac field $(0.45 \mathrm{mT})$ to the sample with frequency $\omega / 2 \pi$ in between $0.01 \mathrm{~Hz}$ and $1.5 \mathrm{kHz}$. The samples had a rather large diamagnetic signal arising from the silicon substrate. This contribution was estimated independently by measuring a bare substrate and found to be linear in field and independent of the temperature. It was subsequently subtracted from all experimental data. Since the mass of deposited Co is accurately known, we give the susceptibility in SI absolute units. Unless indicated otherwise, the data shown in this paper were measured on samples having more than $20 \mathrm{Co} / \mathrm{Al}_{2} \mathrm{O}_{3}$ bilayers in order to maximize their magnetic signals. We have checked for $t_{C o}=0.3 \mathrm{~nm}$ and $t_{C o}=0.7 \mathrm{~nm}$ that the variation of the

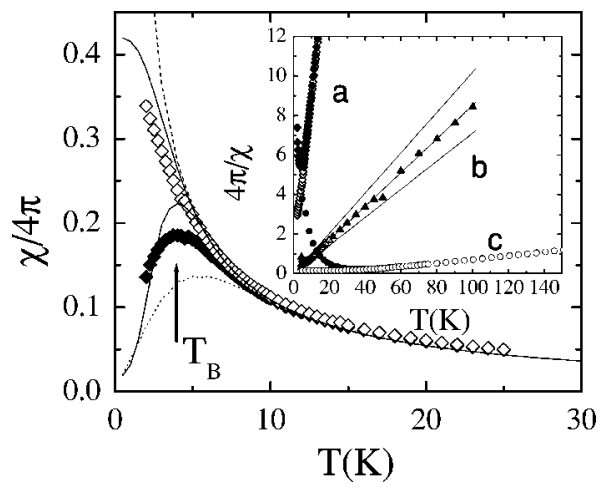

FIG. 1. de susceptibility of a multilayer with $t_{C o}=0.1 \mathrm{~nm}$ and $N=100$ measured with a field of $0.01 \mathrm{~T}$ : open symbols, FC; solid symbols, ZFC. The lines represent the results of calculations performed with the parameters of the size distribution given in Table I: dashed line, equilibrium susceptibility; dotted line, ZFC susceptibility calculated taking $U=K_{e f f} V$ and $K_{e f f}=2.4 \times 10^{6} \mathrm{~J} / \mathrm{m}^{3}$; solid lines, ZFC and FC susceptibilities calculated for surface anisotropy with $K_{s}=0.3 \mathrm{~mJ} / \mathrm{m}^{2}$. Inset: inverse suceptibility of three multilayers: (a) $t_{C o}=0.1 \mathrm{~nm}, N=100$; (b) $t_{C o}=0.3 \mathrm{~nm}, N=40$; and (c) $t_{C o}=0.7 \mathrm{~nm}, N=30$. The lines represent the equilibrium susceptibility of (b) calculated for $\langle D\rangle=1.4 \mathrm{~nm}$ and three values of $\sigma$ : 0.25 (upper curve), 0.3 (medium curve), and 0.35 (lower curve).

magnetization and of the ac susceptibility with temperature and magnetic field is rather insensitive to the precise value of $N$, provided that $N$ is larger than 10 layers.

\section{RESULTS AND DISCUSSION}

\section{A. Superparamagnetic blocking}

The magnetic dc susceptibility was measured by cooling the samples in zero field (ZFC) or in the presence of the measuring magnetic field (FC). Typical ZFC-FC magnetization curves are plotted in Fig. 1. At high temperatures the ZFC and FC curves coincide, indicating that the samples behave as superparamagnets. In this region, both curves follow the Curie-Weiss law $C /(T-\theta)$. The value of $C$ increases as $t_{C o}$ increases (see the inset of Fig. 1), as expected for larger clusters formation as the deposition time of $\mathrm{Co}$ increases. The Curie-Weiss temperature $\theta$ is nearly zero but for the two samples containing the largest particles. This is brought about by the interaction between the particles, which we shall consider in a separate section below. At lower temperature, the two curves start to separate. The ZFC curve shows a maximum at a temperature $T_{B}$ below which the magnetic moments are blocked in fixed directions. It is well known that the phenomenon of blocking is related to the magnetic anisotropy of the particles. ${ }^{18}$ The anisotropy favors some particular orientations of the magnetic moment, two opposite to each other in the simplest case of uniaxial anisotropy, which are separated by activation energy barriers $U$. As the temperature decreases, the number of thermal phonons of energy equal or larger than $U$ decreases, thus leading to an exponential increase of the time $\tau$ needed to reverse the magnetic moment of a particle: ${ }^{18-20}$

$$
\tau=\tau_{0} \exp \left(U / k_{B} T\right)
$$




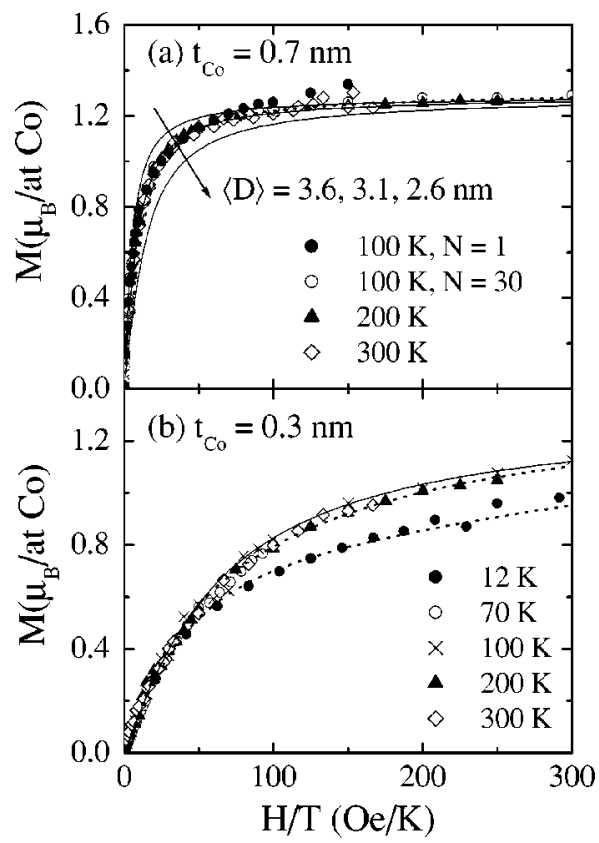

FIG. 2. Equilibrium magnetization of multilayers with $t_{C o}$ $=0.7 \mathrm{~nm}$ (a) and $t_{C o}=0.3 \mathrm{~nm}(\mathrm{~b})$, measured at different temperatures. The lines represent the calculated results. (a) Dotted line, pure Langevin curve for $D=3.1 \mathrm{~nm}$; solid lines, results calculated averaging a Langevin curve over a Gaussian distribution of sizes [see Eq. (2)] with $\sigma=0.2$ and three different values of the average diameter. (b) Solid line, as in the upper picture for $\sigma=0.32$ and $\langle D\rangle=1.4 \mathrm{~nm}$; dotted lines, equilibrium magnetization calculated for $T=12 \mathrm{~K}$ and $T=30 \mathrm{~K}$ with the same size distribution but for uniaxial anisotropy with $U=\pi K_{s} D^{2}$ and $K_{s}=0.2 \mathrm{~mJ} / \mathrm{m}^{2}$.

Here $\tau_{0} \approx 10^{-10}-10^{-13} \mathrm{~s}$ is an inverse attempt frequency, which depends on the damping of the magnetic moment by the phonon or the magnon baths. In this simple picture, the superparamagnetic blocking takes place when $\tau$ equals the measurement time of each experimental point $t_{e}$, thus $T_{B}$ $\simeq \alpha U / k_{B} \ln \left(t_{e} / \tau_{0}\right)$, where $\alpha$ is a constant which depends on the width of the particle size distribution (more details are given below). We have indeed observed that $T_{B}$ increases with the Co deposition time, that is, with the average volume of the aggregates. Therefore, we write $U=K_{e f f} V$, where

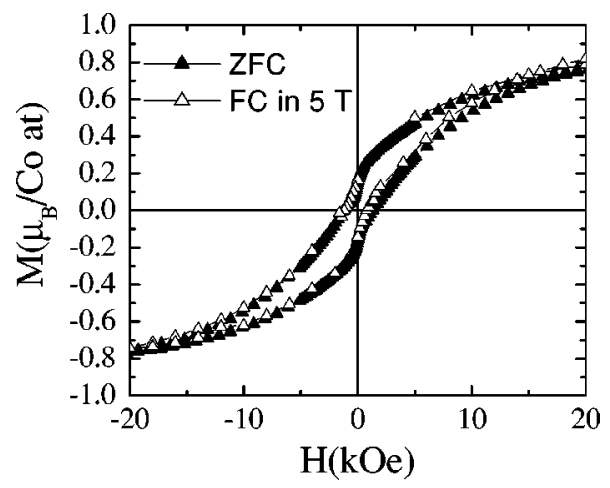

FIG. 3. Hysteresis loop of a multilayer with $t_{C o}=0.1 \mathrm{~nm}$ measured at $T=2 \mathrm{~K}$ after cooling the sample in zero field or in $5 \mathrm{~T}$ from room temperature.

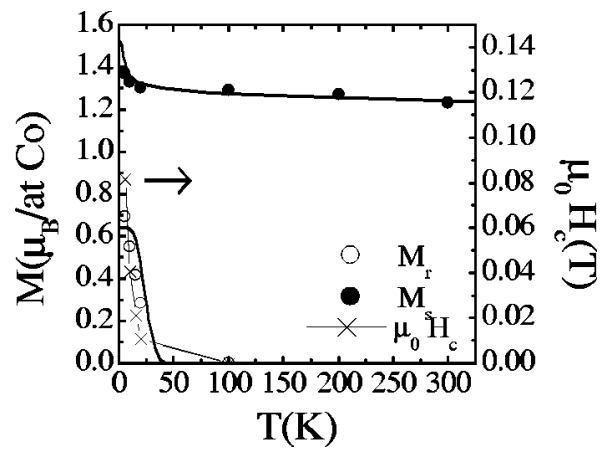

FIG. 4. Left axis: temperature dependence of the remanent magnetization $(\bigcirc)$ and of the saturation magnetization measured with $\mu_{0} H=5 \mathrm{~T}(\bigcirc)$ of a $t_{C o}=0.7 \mathrm{~nm}$ multilayer. The lines are calculated with Eqs. (2) and (9), respectively taking $M_{s b}$ $=1.7 \mu_{B} /$ Co atom, the parameters given in Table I, and the distribution $f(U)$ estimated from the blocking of the ac susceptibility. Right axis: temperature dependence of the coercive field $(X)$ of the same sample.

$K_{\text {eff }}$ is an effective anisotropy constant with contributions from the intrinsic magnetocrystalline anisotropy of the fcc Co and from other sources, such as stress-induced anisotropy or surface-induced anisotropy. The dependence of $K_{e f f}$ on $V$ will be considered below in Sec. IV C.

Above $T_{B}$ the magnetization isotherms are fully reversible because the magnetic moments are in thermal equilibrium. As shown in Fig. 2, the experimental data measured well above the blocking temperature of each sample collapse onto a single curve when they are plotted as a function of $H / T$, indicating that the effect of the anisotropy is weak. Furthermore, pure Langevin curves fit the experimental data reasonably well, which shows that the size distributions of all these samples are narrow. Below $T_{B}$, the magnetization shows hysteresis (see Fig. 3) with both the coercive field $H_{c}$ and the remanence $M_{r}$ increasing as the temperature decreases (cf. Fig. 4).

We plot in Fig. 5 the low- $T$ values of $M_{r}$ and of the

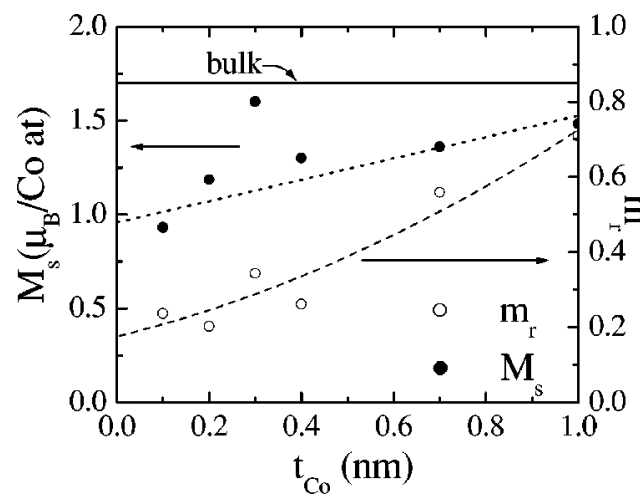

FIG. 5. Variation of the low-temperature reduced remanent magnetization $m_{r}\left(\bigcirc\right.$, right axis) and saturation magnetization $M_{s}(\mathbf{O}$, left axis) with the amount of deposited Co for all samples studied. The dotted line represents $\left(1-x_{\text {para }}\right) 1.7+x_{\text {para }} \mu_{B}$ with $x_{\text {para }}$ as obtained from TEM studies and the dashed line is a guide to the eye. 
saturation magnetization $M_{s}$ as a function of $t_{C o}$. It is interesting to note that, for $t_{C o}<0.7 \mathrm{~nm}$, the reduced remanence $m_{r}=M_{r} / M_{s}$ is smaller than the value $1 / 2$ predicted for particles having their easy axes oriented at random. ${ }^{21}$ We attribute the reduction of $m_{r}$ below this value to the presence of paramagnetic atoms that do not contribute to $m_{r}$. A Curie tail shown by the saturation magnetization at the lowest temperatures is also related to this extra contribution. The excess paramagnetism arises likely from single atoms or very small clusters that are formed in the first stages of the preparation process and which do not give rise to further aggregation. ${ }^{15,16,22}$ It was found that the fraction $x_{\text {para }}$ of Co which is deposited but is not detected by TEM increases as $t_{C o}$ decreases. Accordingly, $m_{r}$ decreases as the amount of deposited Co decreases. On the other hand, the sample with $t_{C o}=1 \mathrm{~nm}$ has $m_{r}=0.71$, that is, larger than $1 / 2$, likely because of the predominant ferromagnetic coupling between particles.

It is also remarkable that the average magnetic moment per atom for the whole sample, as obtained from $M_{s}$ of Figs. 4 and 5 , is smaller than the value for bulk Co $\left(1.7 \mu_{B}\right.$ per Co atom) for all samples and that it decreases as $t_{C o}$ decreases (and thus as the average size of the particles decreases). This dependence is opposite to that observed for free Co clusters in beams ${ }^{8}$ and also for Co particles of similar size supported in a solid matrix. ${ }^{10,23}$ In those experiments, the measured magnetic moment per atom exceeded the bulk value and it was found to increase as the diameter of the particles decreases.

The smaller value of $M_{s}$ that we measure could be related to the presence of an oxide layer at the surface of the particles, which orders antiferromagnetically. However, we did not find any trace of oxide in electron energy loss spectroscopy (EELS) measurements. Moreover, it is known that the exchange interaction between this layer and the magnetic core of the particles would also induce a net anisotropy on the latter. ${ }^{24}$ This so-called exchange anisotropy leads to a shift of the hysteresis loops when the sample is cooled down in the presence of a magnetic field. For example, Peng and co-workers ${ }^{25}$ have recently measured an exchange bias field as large as $1 \mathrm{~T}$ for $\mathrm{CoO}$ coated $\mathrm{Co}$ clusters having a diameter of $6 \mathrm{~nm}$ and $13 \mathrm{~nm}$. By contrast, as we show in Fig. 3, the hysteresis loops measured after cooling the sample in zero field or in $5 \mathrm{~T}$ from room temperature are nearly identical, thus with no evidence for an antiferromagnetic order at the surface layer. Thus, we conclude that most of the particles are free from oxidation.

It is, however, still possible that some of the Co atoms, in close contact with the $\mathrm{Al}_{2} \mathrm{O}_{3}$ matrix, have a weak chemical link with it. This chemical bonding can reduce the number of unpaired electrons and then quench the magnetic moment of the metal atom, as was shown by van Leeuwen and co-workers. ${ }^{5}$ From our data, it is not possible to determine whether the atoms involved in the reduction of the average magnetic moment are located at the periphery of the particles or are those atoms which do not form aggregates, because the relative concentration of both increases as the average size of the clusters decreases.
Therefore, in what follows, we approach the problem in a different way. As a starting point of the analysis we consider that the spheres have the bulk magnetization: $M_{s b}=1.7 \mu_{B}$ per Co atom, whereas the missing magnetic moment is exclusively attributed to the paramagnetic Co fraction. The contribution of the clusters to the net saturation magnetization of each sample then equals $\left(1-x_{\text {para }}\right) M_{s b}$. It was obtained by subtracting the low- $T$ paramagnetic tail from the total $M_{s}$. This tail corresponds approximately to $(0.7-1) \mu_{B}$ per paramagnetic atom for all samples, which indicates that these isolated atoms have in average only one unpaired electron. In this way, $x_{\text {para }}$ is also estimated. The so-obtained $x_{\text {para }}$ values (see Table I) increase as $t_{C o}$ decreases and are in reasonably good agreement with the values previously determined by TEM. ${ }^{16}$ We show in Fig. 5 that $\left(1-x_{\text {para }}\right) 1.7$ $+x_{\text {para }} \mu_{B}$ calculated using the TEM values reproduces well the observed dependence of $M_{s}$ as a function of $t_{C o}$ (with the exception of the sample with $\left.t_{C o}=0.3 \mathrm{~nm}\right)$. We also find that the remanent magnetization calculated as $0.5(1$ $\left.-x_{\text {para }}\right) M_{s b}$ accounts for the experimental data for $t_{C o}$ $\leqslant 0.4 \mathrm{~nm}$. Above this thickness, the interaction between the particles becomes important (see Sec. IV D) and, consequently, the remanence becomes larger than what is expected for noninteracting magnetic moments.

\section{B. Determination of the particle size distribution}

In this section we will try to determine the particle size distribution from the equilibrium magnetic properties of each sample and compare it with the results obtained by TEM. In Fig. 2 we have plotted the equilibrium magnetization $M$ of two different samples having $t_{C o}=0.3 \mathrm{~nm}$ and $0.7 \mathrm{~nm}$, respectively. We recall that, for a set of magnetic moments $\mu$ without anisotropy, $M(H, T, \mu)=M_{s} L(\mu H / T)$, where $L$ denotes the Langevin function. If the anisotropy energy is taken into account, there is no analytical expression for $M(H, T, \mu)$ and the shape of the magnetization curve deviates from the pure Langevin form when $U / k_{B} T$ is large. However, it can still be evaluated numerically, as was described in Ref. 26.

For a real sample, we have to average $M(H, T, \mu)$ over the appropriate distribution of particle sizes. Comparing the calculated magnetization to the experimental data we shall try next to get information about this distribution. In order to directly compare our results with those obtained previously by TEM, we define $g(D)$ as the distribution of number of particles having a diameter equal to $D$. For spherical particles $\mu=\pi M_{s b} D^{3} / 6$, where $M_{s b}$ is, for the reasons given in the previous section, taken as equal to the saturation magnetization of bulk Co. We fit the experimental data using the following expression:

$$
\begin{aligned}
M(H, T)= & x_{\text {para }} \mu_{B} \tanh \left(\frac{\mu_{B} H}{k_{B} T}\right)+\left(1-x_{\text {para }}\right) \\
& \times M_{s b} \frac{\int g(D) V\left[M(H, T, \mu) / M_{s}\right] d D}{\int g(D) V d D},
\end{aligned}
$$


taking for $g$ a Gaussian distribution.

For each sample, we fit only data measured at temperatures for which the two calculations, with and without anisotropy, give approximately the same result. We give an example of this method in the lower panel of Fig. 2 for a multilayer with $t_{C o}=0.3 \mathrm{~nm}$. Above $30 \mathrm{~K}$, the calculations performed with and without anisotropy almost coincide. For lower temperatures, close to $T_{B}=8.6 \mathrm{~K}$, the experimental magnetization starts to deviate from the pure isotropic behavior, as happens for the data measured at $T=12 \mathrm{~K}$. Even then, the experimental data are rather well reproduced by our calculations if we use the value of $K_{\text {eff }}$ determined from the blocking of the ac susceptibility (see Sec. IV C below). $\langle D\rangle$ values obtained from the fit for all samples are given in Table I. We find that $\langle D\rangle$ increases with $t_{C o}$, as expected. For most of the samples it is, however, larger by $10 \%-40 \%$ than the values that were previously found by TEM. This discrepancy can be ascribed to the fact that the TEM experiments were performed on single layers deposited on a special carbon substrate, whereas we have measured multilayers prepared on a Si oxide. However, we have also measured a monolayer with $t_{C o}=0.7 \mathrm{~nm}$ and obtained almost the same magnetization results (see the upper panel of Fig. 2) as for a multilayer. An alternative explanation is that the saturation magnetization of the smallest particles is enhanced with respect to the bulk, as was found in similar systems of Co clusters. ${ }^{7,8,10,23}$ However, even if we had used the maximum value of $2.3 \mu_{B}$ per Co atom, which was found by Respaud et al., $\langle D\rangle$ would have decreased by $10 \%$ only, that is, within the uncertainty of the fitting procedure. In order to get the same diameters that were observed by TEM, $M_{s}$ should be as large as $3 \mu_{B}-4 \mu_{B}$ per Co atom for the smallest clusters.

The fit of the magnetization curves is more sensitive to the value of $\langle D\rangle$ than to the width of the distribution $\sigma$. In fact, it is possible to obtain a reasonably good fit by using a single Langevin curve $(\sigma=0)$ with almost the same $\langle D\rangle$ value as for $\sigma \neq 0$ (see Fig. 2, upper panel). In order to get a better estimation of $\sigma$, we have also fitted the equilibrium susceptibility, as obtained from the low field dc or ac measurements well above $T_{B}$, using the following expression:

$$
\chi_{e q}=\frac{\int g(D) V\left(M_{s b}^{2} V / 3 k_{B} T\right) d D}{\int g(D) V d D} .
$$

This formula is valid also for particles with uniaxial magnetic anisotropy if, as is the case for our samples, the anisotropy axes are not oriented. ${ }^{27}$ It turns out that the equilibrium susceptibility is very sensitive to the presence of large particles in the distribution and therefore to $\sigma$, as is shown in the inset of Fig. 1. For this reason, the contribution of the paramagnetic moments to $\chi_{e q}$ can be neglected in all cases. The values for $\langle D\rangle$ and $\sigma$ that are given in Table I are those which reproduce best both the equilibrium magnetization isotherms and the equilibrium susceptibility. The width of the distribution is found to be rather constant and in good agreement with the value found previously by TEM. The slight increase of $\sigma$ as the average size of the aggregates decreases was also observed in the TEM data. ${ }^{15}$ In conclusion, the codeposition of $\mathrm{Co}$ and $\mathrm{Al}_{2} \mathrm{O}_{3}$ yields Co clusters of controllable size and with a narrow and nearly constant distribution of diameters.

\section{Magnetic anisotropy}

Next, we want to study the dependence of the effective anisotropy on the cluster size. The anisotropy of the particles can be estimated by comparing the average activation energy $\langle U\rangle$ to the average volume $\pi / 6\langle D\rangle^{3}$. Usually $\langle U\rangle$ is estimated as $\langle U\rangle=25 k_{B} T_{B}$, where $T_{B}$ is the temperature of the maximum of the ZFC susceptibility. However, this procedure leads to an overestimation of the anisotropy because $T_{B}$ depends not only on $\langle U\rangle$ but also increases with $\sigma{ }^{28,29}$

In order to get more reliable values we need a way to obtain the full distribution of activation energies $f(U)$ and then to find which value of $U$ corresponds to particles with a diameter equal to $\langle D\rangle$. Fortunately, $f(U)$ can be directly determined from ac susceptibility data measured near the superparamagnetic blocking temperature $T_{B} \cdot{ }^{29,30}$ As mentioned above, the blocking occurs when the average relaxation time becomes of the order of $1 / \omega$. It is therefore clear that the temperature dependence of $\chi^{\prime}$ and $\chi^{\prime \prime}$ near $T_{B}$ is determined by the distribution of $U$ among the particles. In order to relate $\chi^{\prime}$ and $\chi^{\prime \prime}$ to the distribution $f(U)$ it is common to assume that those particles having $U>U_{b}$ are fully blocked and the ones that do not fulfill this condition are in equilibrium. This hypothesis is reasonable because the relaxation time depends exponentially on $U$ according to Eq. (1). For noninteracting particles,

$$
\begin{gathered}
\chi^{\prime} \simeq \int_{0}^{U_{b}} \chi_{e q}(U, T) f(U) d U+\frac{2}{3} \int_{U_{b}}^{\infty} \chi_{\perp}(U, T) f(U) d U \\
\chi^{\prime \prime} \simeq \frac{\pi}{2} k_{B} T \chi_{e q}\left(T, U_{b}\right) f\left(U_{b}\right)
\end{gathered}
$$

where $U_{b}=k_{B} T \ln \left(1 / \omega \tau_{0}\right)$ is the activation energy of those particles having exactly $\tau=t_{e}$ at a given temperature. $\chi_{e q}$ $=M_{s b}^{2} V / 3 k_{B} T$ and $\chi_{\perp}=M_{s b}^{2} / 2 K_{\text {eff }}$ are the equilibrium susceptibility and the reversible (high-frequency limit) susceptibility, respectively. ${ }^{27}$ It follows from Eq. (5) that $U f(U)$ can be directly determined by plotting $\chi^{\prime \prime}$ versus the scaling variable $U_{b}$. In Fig. 6 we show the result for a multilayer with $t_{C o}=0.7 \mathrm{~nm}$. Similar results were obtained for the other samples.

It is important to note here that $f(U)$ is the fraction of the total magnetic volume occupied by particles having the activation energy $U$, since the susceptibility is mainly dominated by the contribution of the largest particles. Contrary, $g(D)$ gives instead the number of particles of a given size. The two distributions are related as follows:

$$
f(U)=\frac{V g(D)}{(d U / d D) \int_{0}^{\infty} V g(D) d D} .
$$




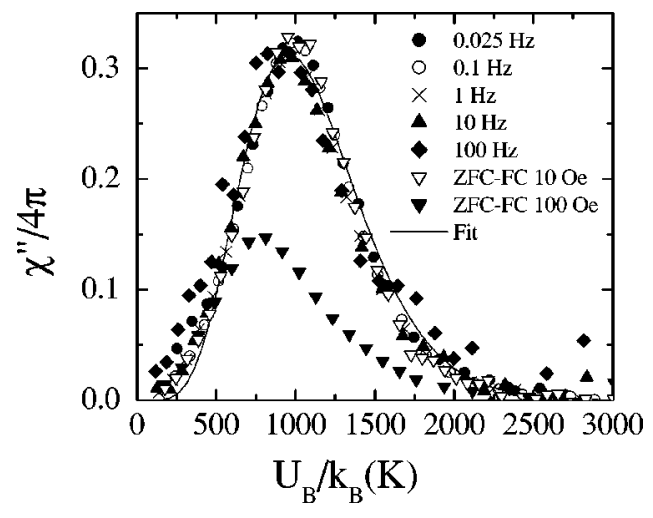

FIG. 6. Imaginary part of the susceptibility of a multilayer with $t_{C o}=0.7 \mathrm{~nm}$ and $N=30$ plotted as a function of the scaling variable $U_{B} / k_{B}=T \ln \left(1 / \omega \tau_{0}\right)$, with $\tau_{0}=10^{-13}$ seconds. The solid line is a fit according to Eq. (5) taking a Gaussian for $g(D)$. Results obtained as explained in the text [cf. Eq. (7)] from ZFC-FC magnetization curves measured with two different magnetic fields are also shown for comparison.

For spherical particles $U(D)=K_{e f f}(\pi / 6) D^{3}$. Therefore $f(U)$ is, apart from normalization factors, proportional to $\left(U / K_{e f f}\right)^{1 / 3} g\left[\left(6 U / \pi K_{e f f}\right)^{1 / 3}\right]$. Using this relationship and taking a Gaussian $g(D)$, it is possible to fit $\chi^{\prime \prime}\left(U_{b}\right)$. The anisotropy constant is then simply the ratio between $U(\langle D\rangle)$ and $V(\langle D\rangle)$. Although the fit is rather good, we find that the function $g$ that is extracted in this way from $f(U)$ (or $\chi^{\prime \prime}$ ) is systematically narrower than the size distribution obtained previously using the equilibrium magnetization and magnetic susceptibility. We will discuss later on the possible physical origin of this discrepancy.

The distribution $f(U)$ can also be obtained by a different method, which makes use of the ZFC and FC dc susceptibility curves measured at low enough magnetic fields. The difference between the ZFC and FC magnetization curves stems from the different contributions of the blocked particles to each of them. Neglecting the weak variation of $M_{s b}$ with $T$, this contribution only depends on $T$ via the critical energy $U_{b}$ which determines the relative number of blocked and superparamagnetic particles at a given temperature. Using the same approximation which led to Eqs. (4) and (5) for the ac susceptibility, it is possible to show that

$$
\frac{\partial\left(M_{F C}-M_{Z F C}\right)}{\partial T}=-M_{i r r}\left(U_{b}, T, H\right) f\left(U_{b}\right),
$$

where $M_{i r r}=M_{e q}-M_{r e v}$ and $M_{r e v}$ is the magnetization brought by the reversible rotation of the magnetic moments. This expression is valid provided that the applied magnetic field is much smaller than the anisotropy field $H_{k}$ $=2 K_{\text {eff }} / M_{s b}$, as is actually the case in our experiments. It is also possible to approximate $M_{i r r}\left(U_{b}\right) \simeq \chi_{e q}\left(U_{b}\right) H$. Therefore, Eq. (7) gives an independent method to determine $f(U)$. We plot in Fig. 6 the results obtained for an applied field of $0.001 \mathrm{~T}$, which are in good agreement with the ac susceptibility data. In the same figure, data obtained for $\mu_{0} H=0.01 \mathrm{~T}$ are also shown. In this case the maximum of the distribution shifts towards lower values of $U_{b}$, indicating

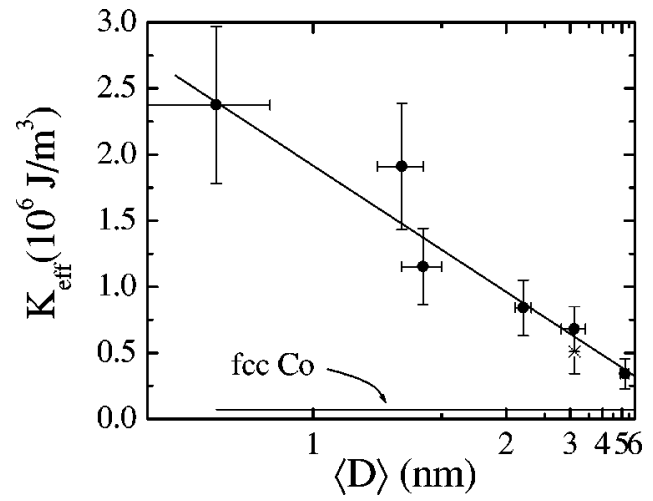

FIG. 7. Size dependence of the effective anisotropy constant for all samples investigated. $\times$ gives the value obtained for a singlelayer with $t_{C o}=0.7 \mathrm{~nm}$. The solid line is a best squares fit of the data to Eq. (8).

that the activation energy decreases in a magnetic field. In addition, the distribution function broadens a bit as a result of the random orientation of the easy axes.

We now come back to our main goal. The anisotropy constant is plotted in Fig. 7 as a function of the average diameter of the aggregates. It is interesting to compare these experimental data with the constant $K_{\text {eff }}$ that is estimated using only the intrinsic magnetocrystalline anisotropy of bulk Co. For hcp Co, the stable phase for large particles, $K_{\text {eff }}$, equals the intrinsic uniaxial anisotropy constant $K$ $=4.3 \times 10^{5} \mathrm{~J} / \mathrm{m}^{3}$. However, the structural characterization of all samples studied here shows that they crystallize in the fcc phase. Therefore, we would expect that the intrinsic anisotropy of the particles in our samples would be smaller than that for hcp Co. For cubic anisotropy ${ }^{28,31} K_{e f f}=K / 4$, where $K$ is the second-order intrinsic anisotropy constant. Taking $K=2.6 \times 10^{5} \mathrm{~J} / \mathrm{m}^{3}$ for $\mathrm{fcc} \mathrm{Co},{ }^{10,32}$ this gives $K_{\text {eff }} \simeq 6.5$ $\times 10^{4} \mathrm{~J} / \mathrm{m}^{3}$. Therefore, the values that we find for all samples are almost one to two orders of magnitude larger than expected for magnetocrystalline anisotropy. Furthermore, $K_{\text {eff }}$ is observed to increase as $\langle D\rangle$ decreases. The size dependence of the effective anisotropy follows approximately the following phenomenological expression:

$$
K_{e f f}=K_{\infty}+\frac{6 K_{s}}{\langle D\rangle}
$$

with $K_{\infty}=5( \pm 2) \times 10^{4} \mathrm{~J} / \mathrm{m}^{3} \quad$ and $\quad K_{s}=3.3( \pm 0.5)$ $\times 10^{-1} \mathrm{~mJ} / \mathrm{m}^{2}$. This result is robust in the sense that it does not change qualitatively if we use the average diameter found by TEM, instead of the values obtained from the magnetization data. The first term is close to $K / 4$ and can therefore be identified as the contribution of the intrinsic anisotropy. The second one is proportional to the fraction of atoms located at the periphery of the particles, which can be more than $80 \%$ of all Co atoms for the smallest clusters studied here. Our experimental results indicate then that there exists a rather large contribution of the surface atoms to the net anisotropy.

The enhancement of the magnetic anisotropy of nanometer-sized metallic particles with respect to the bulk 
has been previously reported by several authors. ${ }^{9,10,23}$ For Co particles with diameters varying between 4.4 and $1.8 \mathrm{~nm}$, Chen and co-workers ${ }^{10}$ obtained $K_{\text {eff }}$ which increases from about $5 \times 10^{5} \mathrm{~J} / \mathrm{m}^{3}$ to about $3 \times 10^{6} \mathrm{~J} / \mathrm{m}^{3}$. These values are even larger than ours. However, they are of the same order as the values that would have been obtained if we had used the temperature of the maximum of the ZFC susceptibility, as was done by the authors. More recently, Respaud et al. ${ }^{23}$ studied the anisotropy of Co particles of 1.5 and $1.9 \mathrm{~nm}$ by fitting the whole ZFC and FC magnetization curves, a method that can be considered as equivalent to ours. They found $K_{\text {eff }} \simeq 8.3 \times 10^{5} \mathrm{~J} / \mathrm{m}^{3}$ and $K_{\text {eff }} \simeq 7.3 \times 10^{5} \mathrm{~J} / \mathrm{m}^{3}$, respectively, in reasonably good agreement with our data. The existence of a large surface anisotropy in metallic particles is thus well established experimentally.

The origin of this extra anisotropy has been related to the modification of the electrostatic and exchange interactions of the atoms located at the surface, ${ }^{4,33,34}$ which depends largely on whether the surface is oxidized or not. Since our samples show no oxide layer, we have to consider how the properties of a "bare" metallic surface are modified with respect to the bulk. The value of $K_{s}$ that we have found is actually comparable to the perpendicular anisotropy measured in free Co surfaces. ${ }^{35}$ It is commonly accepted that this perpendicular anisotropy is related to the appearance of a large orbital magnetic moment on these atoms. ${ }^{36}$ The $3 d$ electrons become more localized at the surface and, consequently, have larger orbital moments. The same theoretical interpretation can be applied to the atoms at the periphery of small metallic clusters. ${ }^{4}$ In this case, the enhanced anisotropy at the surface extends to the inner atoms via the strong exchange interaction with them, which leads to an increase of the average anisotropy of even spherical clusters. ${ }^{37}$ This interpretation has been confirmed by $\mathrm{x}$-ray magnetic dichroism experiments performed on $\mathrm{Au} / \mathrm{Co} / \mathrm{Au}$ layers ${ }^{35}$ and more recently also on Co-disk-like aggregates supported on Au surfaces. ${ }^{11}$ It was found that the orbital component $m_{L}$ of the total magnetic moment scales with the fraction of atoms located at the surface of the aggregates. For spherical clusters, as the ones studied here, we expect then that $m_{L} \propto 1 /\langle D\rangle$, dependence that we have indeed observed for $K_{\text {eff }}$. We therefore conclude that the observed increase of $K_{\text {eff }}$ is likely due to the increasingly localized character of the $3 d$ electrons of the atoms located at the surface.

Once the particle size distribution and the anisotropy are known, it is possible to predict the time-dependent magnetic response of the samples and compare it to the experiment. Examples of these calculations are compared to the experimental data in Figs. 1, 2, and 4. The calculations account very well for the experimental data measured above $T_{B}$, as expected. They also reproduce in Fig. 1 the deviation of the FC susceptibility from the equilibrium susceptibility that takes place below $5 \mathrm{~K}$. However, they reproduce neither the position nor the shape of the maximum of the ZFC susceptibility. Another example of this discrepancy is shown in Fig. 8 , where we plot the experimental $\chi^{\prime}$ for a multilayer with $t_{C o}=0.3 \mathrm{~nm}$ and the values calculated (dotted line) with Eq. (4). Again, the width of the blocking transition is clearly overestimated by the calculations.

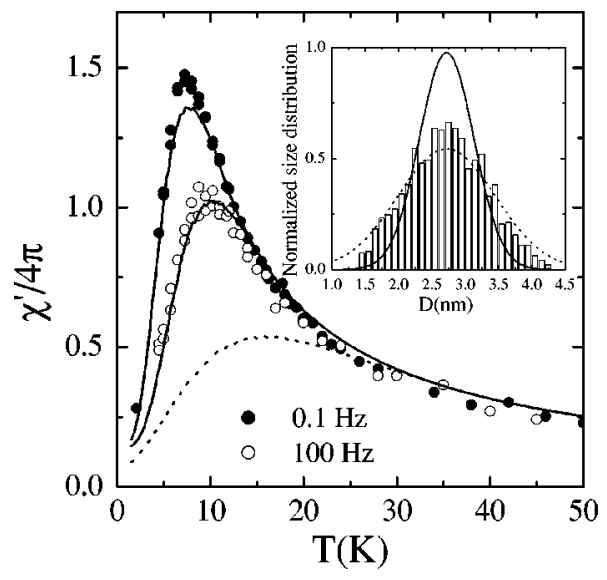

FIG. 8. Real part of the susceptibility of a Co multilayer with $t_{C o}=0.3 \mathrm{~nm}$ and $N=40$ measured for two different frequencies. The dotted line is calculated for $\omega / 2 \pi=0.1 \mathrm{~Hz}$ with Eq. (4) using the parameters of Table I and taking $U=\pi K_{e f f} D^{3} / 6$, with $K_{\text {eff }}$ $=1.15 \times 10^{6} \mathrm{~J} / \mathrm{m}^{3}$. The solid lines are calculated taking $U$ $=\pi K_{s} D^{2}$, with $K_{s}=2 \times 10^{-1} \mathrm{~mJ} / \mathrm{m}^{2}$. Inset: the size distribution determined by TEM for $t_{C o}=0.7 \mathrm{~nm}$ is compared to the distributions obtained from $\chi^{\prime \prime}$ for two limiting cases where the anisotropy is either dominated by the intrinsic (volume) contribution (solid line) or by the surface anisotropy (dotted line). The scaling in the horizontal axis gives respectively $K_{e f f}=10^{6} \mathrm{~J} / \mathrm{m}^{3}$ and $K_{s}$ $=0.33 \mathrm{~mJ} / \mathrm{m}^{2}$.

We recall here that we have found that the activation barrier distribution is systematically narrower than the size distribution for all samples. As an example, in the inset of Fig. 8 we plot the size distribution $g(D)$ of a multilayer with $t_{C o}=0.7 \mathrm{~nm}$ extracted from $\chi^{\prime \prime}$ and directly observed by TEM. The horizontal scale for the former distribution is $\left(6 U_{b} / \pi K_{e f f}\right)^{1 / 3}$, with $K_{e f f}=10^{6} \mathrm{~J} / \mathrm{m}^{3}$. It is tempting now to attribute the "narrowing" of the blocking transition to the effect of the surface anisotropy. When $K_{s} / D \gg K_{\infty}$ then $U$ $\approx K_{s} S$, where $S=\pi D^{2}$ is the surface of the particle. It follows then from Eq. (6) that $f(U) \propto D^{2} g(D)$ and the width of the distribution of activation energies must then be smaller than when $U \propto V$. Figure 8 shows indeed that when the same susceptibility data are represented versus the variable $\left(U_{b} / \pi K_{s}\right)^{1 / 2}$ the ensuing size distribution is in better agreement with what it is found by TEM or from the equilibrium magnetization and susceptibility. In this way, we also obtain $K_{s}$ which turns out to be between 0.2 and $0.3 \mathrm{~mJ} / \mathrm{m}^{2}$ for all samples. This value can be then used to recalculate the ac susceptibility and the ZFC magnetization. We find that the calculations performed with the same parameters $\sigma$ and $\langle D\rangle$ as before (see Table I) but taking $U \propto D^{2}$ are in much better agreement with the experiment (see Figs. 1 and 8). Although the width of the of size distribution is not always accurately determined, it seems that the influence of the surface anisotropy also modifies the shape of the susceptibility peak at the blocking. We conclude that the dynamical response of very small particles is therefore determined by the special physical properties of the atoms which are located at their surface. 


\section{Influence of the number of layers: Dipole-dipole interaction between the particles}

There has been some debate during the last years about the effect that the dipole-dipole interaction between magnetic nanoparticles has on their relaxation times. Shtrikman and Wolfarth $^{38}$ and later Dormann et al. ${ }^{39}$ predicted that the effective activation energy increases by an amount that depends on the number and spatial arrangement of the neighbor particles. By contrast, in the model proposed by Mørup and Tronc $^{40}$ the interaction between the particles leads to a lower $U$. The experimental validation of one of these two models is complicated because, for some preparation methods, it is difficult to vary the density of particles in the sample without modifying the distribution of particle sizes. ${ }^{39,41}$

The preparation method of our samples presents a number of advantages. We have seen that the average size can be controlled by changing the deposition time, but also the packing of the particles can be controlled. The TEM images show that the clusters in a layer do not agglomerate and, furthermore, that the deposition of several layers of Co and $\mathrm{Al}_{2} \mathrm{O}_{3}$ leads to a self-organized spatial arrangement of the particles (see Ref. 17). For a multilayer each cluster has, on average, six nearest neighbors in the same plane, three above and another three below it. For $t_{C o}=0.7 \mathrm{~nm}$, the average distance between nearest Co clusters in the same layer is $\Lambda_{\|} \simeq 5.4 \mathrm{~nm}$, whereas the distance to nearest neighbors in adjacent layers is $\lambda=4.5 \mathrm{~nm} .{ }^{17}$ In this section, we compare the relaxation rate of two samples having both $t_{C o}$ $=0.7 \mathrm{~nm}(\langle D\rangle \simeq 3 \mathrm{~nm})$, but very different number of layers, namely, 30 and only one. By going from a monolayer to a multilayer we certainly expect that the average energy of interaction of a particle with the others changes. The interaction energy between particles in adjacent layers is the largest and of the order of $\mu^{2} / \lambda^{3} \approx 40 \mathrm{~K}$. By contrast, in a sample with a single layer, each particles has, in average, only six neighbors coupled by a weaker interaction $\left(\mu^{2} / \Lambda_{\|}^{3} \approx 20 \mathrm{~K}\right)$.

In order to attribute any difference between the two samples to the effect of the interparticle interactions, it is very important to check beforehand that the sizes of the aggregates are the same in both. We showed in Fig. 2 that the equilibrium magnetization curves of the two samples are almost identical, and we compare in Fig. 9 the inverse of their ac susceptibility curves. Above $T_{B}$, the susceptibility follows the Curie-Weiss law, with identical values of $C$, which confirms that $\langle D\rangle$ and $\sigma$ are practically the same. By contrast, the Curie-Weiss temperature $\theta$ is about 2 times smaller for the monolayer, indicating that the average interparticle interaction is notably reduced. It is also apparent that the blocking temperature of the monolayer is smaller than that of the multilayer. As we have done before, the activation energy of the two samples can be compared by plotting $\chi^{\prime \prime}$ measured at different frequencies as a function of the scaling variable $U_{b}$, as shown in the inset of Fig. 9. The maximum of the curve for the monolayer is clearly shifted towards lower values of $U_{b}$ with respect to the maximum obtained for the multilayer. Our data give strong evidence that the interaction between the aggregate layers tends to increase the activation

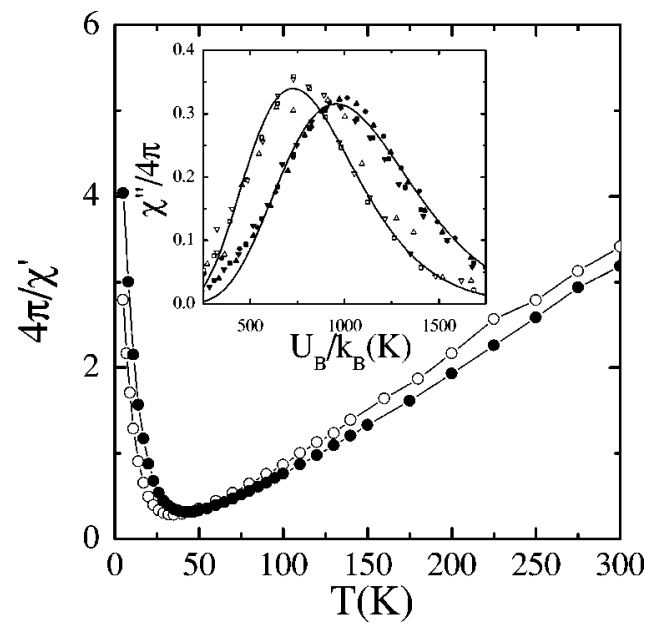

FIG. 9. Inverse ac susceptibility ( $\omega / 2 \pi=1 \mathrm{~Hz})$ of two samples with the same $t_{C o}=0.7 \mathrm{~nm}$ but different number of layers: $\bullet, N$ $=30 ; \bigcirc, N=1$. Inset: for the same two samples, scaling plot of $\chi^{\prime \prime}$ measured at different frequencies: Dots, $0.025 \mathrm{~Hz}$; up triangles, 0.1 $\mathrm{Hz}$; squares, $1 \mathrm{~Hz}$; down triangles, $10 \mathrm{~Hz}$. In both cases $\tau_{0}$ $=10^{-13} \mathrm{~s}$.

energy of each particle, by an amount of about $200 \mathrm{~K}$. This difference is of the same order of magnitude as the interaction energy with the six nearest neighbors in the multilayer. We also find that the relative width of $U f(U)$ has the same value for the two samples, which confirms again that the distribution of particle sizes is the same.

Finally, we would like to mention that the effect that the interaction between the layers has on the effective anisotropy is smaller, by more than one order of magnitude, than the variation of $K_{\text {eff }}$ with size, as shown in Fig. 7. The interaction is, moreover, expected to be much weaker for the smaller particles, in agreement with the small value of $\theta$ found for $t_{C o}<0.7 \mathrm{~nm}$. Therefore, this effect only introduces a small uncertainty (about $5 \%$ ) in the value of $K_{s}$.

\section{E. Magnetic relaxation at low temperatures}

In the previous sections, the reversal of the magnetic moments has been treated as a classical process assisted by the interaction with a thermal bath. However, taken as a quantum variable, the spin of a magnetic cluster $S_{c}=M_{s} V / g \mu_{B}$ can in principle flip also by quantum tunneling across the barrier if the effective Hamiltonian contains terms which deviate from the uniaxial symmetry. ${ }^{14}$ This possibility is very attractive because it would show the existence of quantum effects at the intermediate scale between the microscopic and the macroscopic worlds. Quantum relaxation can dominate over the thermal activation at very low temperatures, when the thermal population of the first excited state doublet $\pm(S-1)$ becomes negligible, and should lead to a saturation of the relaxation rate to a nearly-temperature-independent value. ${ }^{42}$ Such a saturation has indeed been observed in some systems of single-domain particles in the past. ${ }^{13,14,25,43}$

In this section, we present measurements of the relaxation of the remanent magnetization of an initially saturated sample. We have chosen the sample with the smallest Co 


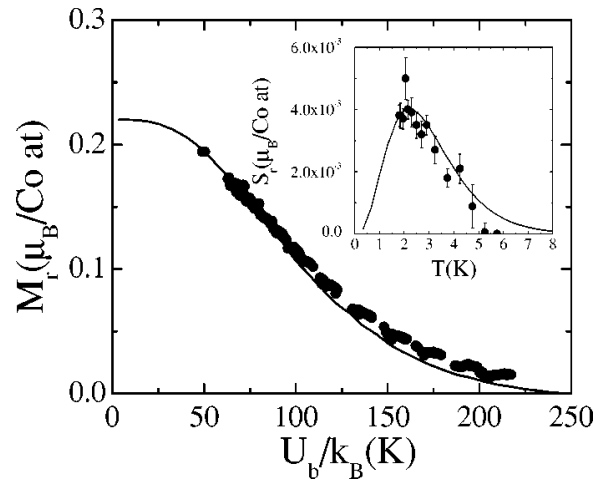

FIG. 10. Time-dependent remanent magnetization of a $\left(\mathrm{Co} 0.1 \mathrm{~nm} \mathrm{Al} \mathrm{O}_{3} 3 \mathrm{~nm}\right)_{100}$ multilayer plotted as a function of the scaling variable $U_{B} / k_{B}=T \ln \left(t_{e} / \tau_{0}\right)$ with $\tau_{0}=10^{-13} \mathrm{~s}$. The inset shows the temperature dependence of the magnetic viscosity. The solid lines are calculated according to Eqs. (9) and (10).

clusters for two reasons: first, because the rate for quantum relaxation must be the largest for these clusters of only about 25-50 atoms, and second, because this sample shows the strongest anisotropy. The separation of the two lowest-lying state doublets, which is roughly given by $\Omega_{0} \approx g \mu_{B} H_{k}$, is then about $3 \mathrm{~K}$, thus larger than the lowest temperature that our magnetometer can reach $\left(T_{\min }=1.7 \mathrm{~K}\right)$. We have measured the decay of the magnetization of the sample that takes place after a magnetic field of $5 \mathrm{~T}$ is switched off at different temperatures. The decay of $M_{r}$ is approximately logarithmic in time. An important advantage of recording the relaxation at zero field is that it can then be easily calculated using our knowledge of the activation energies distribution. At zero field, the equilibrium magnetization is zero for all particles. Therefore, using the same approximation as before, the time-dependent magnetization is given by

$$
M(t, T)=\frac{M_{s}}{2} \int_{U_{b}}^{\infty} f(U) d(U),
$$

where we have made the reasonable approximation that the magnetic moments of the particles are initially saturated by the magnetic field. The factor of $1 / 2$ arises from the reversible rotation of the magnetic moments for a random orientation of the easy axes, as in the Stoner-Wolhfarth model. ${ }^{21}$ As pointed out by Labarta et al. ${ }^{44}$ if the magnetic moments flip by a thermally activated process, the relaxation curves measured at different temperatures should scale when plotted as a function of $U_{b}$. This plot also gives a picture of the relaxation at very long times, which are not experimentally accessible. Our experimental data, which we plot in Fig. 10 do indeed show a rather good scaling for the same $\tau_{0}$ $=10^{-13} \mathrm{~s}$ that was obtained from the shift of the maximum of $\chi^{\prime \prime}$ with frequency. The solid line in the figure was calculated with Eq. (9) using the distribution $f(U)$ that we determined with the method described in Sec. IV C. The scaling of the data confirms that the relaxation mechanism is classical (not tunneling) down to $T=1.7 \mathrm{~K}$.
In the inset of Fig. 10 we show the temperature dependence of the so-called magnetic viscosity $S_{r}$, determined as the slope of the $M_{r}$ vs $\ln (t)$ curves. Below about $2.5 \mathrm{~K}, S_{r}$ does not vary much with $T$. We note, however, that, according to Eq. (9), the magnetic viscosity is just

$$
S_{r} \equiv \frac{\partial M}{\partial \ln t}=-k_{B} T \frac{M_{s}}{2} f\left(U_{b}\right)
$$

and it is therefore proportional to $f(U)$. The apparent saturation of $S$ measured between $1.7 \mathrm{~K}$ and $2.5 \mathrm{~K}$ just reflects the shape of the distribution $f(U)$, and it is indeed rather well described by the "classical" calculation. These data give an example of how important it is to have information about $f(U)$ in order to adequately interpret the relaxation data. $^{45}$

\section{FINAL REMARKS AND CONCLUSIONS}

We have presented a detailed and extensive study of the magnetic properties of Co aggregates prepared by sequential deposition of $\mathrm{Co}$ and $\mathrm{Al}_{2} \mathrm{O}_{3}$. This preparation method enables us to control both the average size and the number of layers independently. We have shown that the distribution of activation energies can be accurately determined from ac susceptibility and ZFC-FC magnetization measurements. We have investigated the variation of the effective anisotropy as the size of the aggregates decreases from about $5 \mathrm{~nm}$ to below $1 \mathrm{~nm}$. We find that $K_{\text {eff }}$ scales with the fraction of atoms located at the periphery of the aggregates. The strength of the surface anisotropy is of the same order of what is found for free Co surfaces and we therefore attribute it to the increase of the orbital magnetic moment of these atoms. Furthermore, the activation energies distribution resembles the distribution of particle's surfaces rather than the volume distribution. For such small clusters, it is therefore more appropriate to write $U=K_{s} S$ than the "traditional" $U$ $=K_{\text {eff }} V$. Using the distributions of sizes and of activation energies that we have determined, we are able to give a quantitative account of all the equilibrium and timedependent experimental quantities. We have also shown that the activation energy increases when the average number of nearest neighbors per particle increases, in agreement with the model of Dormann et al. Finally, the decay of the remanent magnetization of clusters containing only about $25-50$ atoms is shown to proceed via a thermally activated mechanism down to the lowest temperatures investigated.

\section{ACKNOWLEDGMENTS}

We would like to thank Dr. C. Paulsen and Dr. J. Carrey for assistance with some of the experiments reported in this work. This work has been partly funded by Spanish Grant No. MAT 99/1142 and the European ESPRIT contract "MASSDOTS." 
* To whom all correspondence should be addressed. E-mail address: barto@posta.unizar.es

†Present address: Laboratoire de Nanostructures et Magnétisme, DRFMC/SP2M, CEA, 38054 Grenoble Cedex 9, France.

${ }^{1}$ J.L. Dormann, D. Fiorani, and E. Tronc, Adv. Chem. Phys. 98, 283 (1997).

${ }^{2}$ P.V. Hendriksen, S. Linderoth, and P.-A. Lindgard, J. Magn. Magn. Mater. 104-107, 1577 (1992).

${ }^{3}$ B.V. Reddy, S.N. Khanna, and B.I. Dunlap, Phys. Rev. Lett. 70, 3323 (1993).

${ }^{4}$ G.M. Pastor, J. Dorantes-Dávila, S. Pick, and H. Dreyssé, Phys. Rev. Lett. 75, 326 (1995).

${ }^{5}$ D.A. van Leeuwen, J.M. van Ruitenbeek, L.J. de Jongh, A. Ceriotti, G. Pacchioni, O.D. Hüberlen, and N. Rösch, Phys. Rev. Lett. 73, 1432 (1994).

${ }^{6}$ R.H. Kodama, A.E. Berkowitz, E.J. McNiff, Jr., and S. Foner, Phys. Rev. Lett. 77, 394 (1996).

${ }^{7}$ S. Linderoth and S.N. Khanna, J. Magn. Magn. Mater. 104-107, 1574 (1992).

${ }^{8}$ M.L. Billas, A. Chatelain, and W.A. de Heer, Science 265, 1682 (1994)

${ }^{9}$ F. B $\phi$ dker, S. Mørup, and S. Linderoth, Phys. Rev. Lett. 72, 282 (1994)

${ }^{10}$ J.P. Chen, C.M. Sorensen, K.J. Klabunde, and G.C. Hadjipanayis, Phys. Rev. B 51, 11527 (1995).

${ }^{11}$ H.A. Dúrr, S.S. Dhesi, E. Dudzik, D. Knabben, G. van der Laan, J.B. Goedkoop, and F.U. Hillbrecht, Phys. Rev. B 59, R701 (1999)

${ }^{12}$ K.W. Edmons, C. Binns, S.H. Baker, S.C. Thornton, C. Norris, J.B. Goedkoop, M. Finazzi, and N.B. Brookes, Phys. Rev. B 60, 472 (1999).

${ }^{13}$ Quantum Tunneling of Magnetization, edited by L. Gunther and B. Barbara (Kluwer, Dordrecht, 1995).

${ }^{14}$ E. M. Chudnovsky and J. Tejada, Macroscopic Quantum Tunneling of the Magnetic Moment (Cambridge University Press, Cambridge, England, 1998).

${ }^{15}$ J.L. Maurice, J. Briático, J. Carrey, F. Petroff, L.F. Schelp, and A. Vaurès, Philos. Mag. A 79, 2921 (1999).

${ }^{16}$ J. Briático, J.-L. Maurice, J. Carrey, D. Imhoff, F. Petroff, and A. Vaurès, Eur. Phys. J. D 9, 517 (1999).

${ }^{17}$ D. Babonneau, F. Petroff, J.-L. Maurice, F. Fettar, and A. Vaurès, Appl. Phys. Lett. 76, 2892 (2000).

${ }^{18}$ L. Néel, C.R. Acad. Sci. Paris 228, 664 (1949).

${ }^{19}$ W.F. Brown, Jr., Phys. Rev. 130, 1677 (1963).

${ }^{20}$ W.T. Coffey, D.S.F. Crothers, Yu P. Kalmykov, E.S. Massawe, and J.T. Waldron, Phys. Rev. E 49, 1869 (1994).

${ }^{21}$ E.C. Stoner and E.P. Wohlfarth, Philos. Trans. R. Soc. London, Ser. A 240, 599 (1948).

${ }^{22}$ J. Carrey, J.-L. Maurice, P. Jensen, and A. Vaurès, Appl. Surf. Sci. 164, 48 (2000).

${ }^{23}$ M. Respaud, J.M. Broto, H. Rakoto, A.R. Fert, L. Thomas, B. Barbara, M. Verelst, E. Snoeck, P. Lecante, A. Mosset, J. Osuna,
T. Ould Ely, C. Amiens, and B. Chaudret, Phys. Rev. B 57, 2925 (1998).

${ }^{24}$ A.E. Berkowitz and K. Takano, J. Magn. Magn. Mater. 200, 552 (2000).

${ }^{25}$ D.L. Peng, K. Sumiyama, T. Hihara, and S. Yamamuro, Appl. Phys. Lett. 75, 3856 (1999); D.L. Peng, K. Sumiyama, T. Hihara, S. Yamamuro, and T.J. Konno, Phys. Rev. B 61, 3103 (2000).

${ }^{26}$ M. Hanson, C. Johanson, and S. Mørup, J. Phys.: Condens. Matter 5, 725 (1993).

${ }^{27}$ M.I. Shliomis and V.I. Stepanov, Adv. Chem. Phys. 87, 1 (1994).

${ }^{28}$ J.I. Gittleman, B. Abeles, and S. Bosowski, Phys. Rev. B 9, 3891 (1974).

${ }^{29}$ F. Luis, E. del Barco, J.M. Hernández, E. Remiro, J. Bartolomé, and J. Tejada, Phys. Rev. B 59, 11837 (1999).

${ }^{30}$ F. Luis, J. Bartolomé, J. Tejada, and E. Martínez, J. Magn. Magn. Mater. 157-158, 266 (1996).

${ }^{31}$ C.P. Bean and J.D. Livingston, J. Appl. Phys. 30, 120S (1959).

${ }^{32}$ W.A. Sucksmith and J.E. Thompson, Proc. R. Soc. London, Ser. A 225, 362 (1954).

${ }^{33}$ P. Bruno, Phys. Rev. B 39, R865 (1989).

${ }^{34}$ R.H. Kodama and A.E. Berkowitz, Phys. Rev. B 59, 6321 (1999).

${ }^{35}$ D. Weller, J. Stöhr, R. Nakajima, A. Carl, M.G. Samant, C. Chappert, R. Mégy, P. Beauvillain, P. Veillet, and G.A. Held, Phys. Rev. Lett. 75, 3752 (1995).

${ }^{36}$ Ding-sheng Wang, R. Wu, and A.J. Freeman, Phys. Rev. Lett. 70, 869 (1993).

${ }^{37}$ D.A. Dimitrov and G.M. Wysin, Phys. Rev. B 50, 3077 (1994); 51, 11947 (1995).

${ }^{38}$ S. Shtrikman and E.P. Wholfarth, Phys. Lett. 85A, 467 (1981).

${ }^{39}$ J.L. Dormann, L. Bessais, and D. Fiorani, J. Phys. C 21, 2015 (1988).

${ }^{40}$ S. Mørup and E. Tronc, Phys. Rev. Lett. 72, 3278 (1994).

${ }^{41}$ R.W. Chantrell, G.N. Coverdale, M. El Hilo, and K. O’Grady, J. Magn. Magn. Mater. 157-158, 250 (1996).

${ }^{42}$ N.V. Prokof'ev and P.C.E. Stamp, J. Low Temp. Phys. 104, 143 (1996).

${ }^{43}$ L1. Balcells, J.L. Tholence, S. Linderoth, B. Barbara, and J. Tejada, Z. Phys. B: Condens. Matter 89, 209 (1992); C. Paulsen, L.C. Sampaio, B. Barbara, R. Tucoulou-Tachoueres, D. Fruchart, A. Marchand, J.L. Tholence, and M. Uehara, Europhys. Lett. 19, 643 (1992); J. Tejada, Ll. Balcells, S. Linderoth, R. Perzynski, B. Rigau, B. Barbara, and J.C. Bacri, J. Appl. Phys. 73, 6952 (1993); X.X. Zhang and J. Tejada, ibid. 75, 5637 (1994); R.H. Kodama, C.L. Seaman, A.E. Berkowitz, and B.M. Maple, ibid. 75, 5639 (1994); M.M. Ibrahim, S. Darwish, and M.S. Seehra, Phys. Rev. B 51, 2955 (1995); X.X. Zhang, J.M. Hernández, J. Tejada, and R.F. Ziolo, ibid. 54, 4101 (1996).

${ }^{44}$ A. Labarta, O. Iglesias, Ll. Balcells, and F. Badía, Phys. Rev. B 48, 10240 (1993).

${ }^{45}$ B. Barbara and L. Gunther, J. Magn. Magn. Mater. 128, 35 (1993). 